\title{
A REVIEW OF GALACTIC MILLISECOND PULSAR SEARCHES
}

\author{
A. G. LYNE \\ The University of Manchester, \\ Nuffield Radio Astronomy Laboratories, \\ Jodrell Bank, Macclesfield, \\ Cheshire, SK11 9DL, UK.
}

\begin{abstract}
Since the discovery of the first millisecond pulsar in 1982, the main discoveries of more of these exotic objects have come in two bursts. The first followed the realization late in the decade that they might be formed readily in globular clusters and resulted in the discovery of about two dozen objects in these clusters between 1987 and 1990. The second burst started shortly after this as improved computer technology permitted widespread searches of the Galaxy with high sensitivity and is continuing at the present time. This paper describes the main successful surveys for these galactic millisecond pulsars of which 29 are now known. 21 of these are in circular orbits with low-mass companion stars. The large number of objects now available allows a number of firm statistical trends to be seen which all point to an evolutionary phase of these systems involving the spin-up of old neutron stars during the accretion of matter from less evolved companions during their giant phase. Surveys of the present generation are still very insensitive to short period pulsars or highly accelerated pulsars in close or massive binary systems, limitations which should be removed in future years.
\end{abstract}

\section{Introduction}

In 1982, the first millisecond pulsar (MSP) was discovered by Backer et al. In spite of intense efforts during the next few years, only 2 more had been discovered by 1987. PSR B1953+29 was found by Boriakoff et al. (1983) and PSR B1855+09 by Stokes et al. (1986). At the time, the lack of discoveries suggested that they were rare objects in the sky. 
The main reason for the small number in fact was that the searches were severely limited by the data storage and computer technology available at that time. The problem lies in the dispersion of the pulsed signals by the ionized component of the interstellar medium, preventing the search process being simply a periodicity search of a time sequence. Because the pulses at low frequencies are delayed relative to higher frequencies, recording a single, wide receiver band, which is required to provide high sensitivity, results in narrow pulses being broadened, possibly to such an extent that they cover more than the whole pulse period so that no periodicity would be observed at all. The most effective way of combating this is to split the receiver band into many narrow channels and to record the output of all of these in order to permit subsequent searches over many values of dispersion measure. Higher time resolution requires the use of narrower filters, so that, roughly speaking, in order to cope with dispersion, the size of the data sets and the magnitude of the processing tasks both increase as the square of the sampling rate. Thus, moving from the earlier searches for normal pulsars, which sampled at about $100 \mathrm{~Hz}$, to the millisecond pulsar searches sampling at $3 \mathrm{kHz}$ required 3 orders of magnitude increase in computing resources.

Typical sampling rates in modern searches are $3-4 \mathrm{kHz}$ for each of several hundred frequency channels, amounting to approximately 1 million data samples/second. For instance a 2.5 minute observation of the present Parkes survey described below would result in a data set of 128 million data points to be stored and processed. It would have taken in excess of one day to process such a dataset on a Vax 780 , the standard computer available to astronomers 10 years ago. The small number of millisecond pulsars that were found in these early years were mostly discovered with the aid of super computers.

Because of these limitations, large scale surveys of the sky were impossible in the 1980s. However, it was realised that the origin of the high rotation rate of MSPs might lie in the spin up of old, ordinary pulsars during mass transfer in the accretion of material from a less evolved companion star in its giant phase (Fabian, Pringle \& Rees 1975; Alpar et al. 1982). Such objects are believed to be witnessed in the low-mass X-ray binary systems (LMXBs). The large number of LMXBs seen in globular clusters suggested the possibility of many spun-up neutron stars in these clusters. Subsequently, in 1987, the first MSP was found in the core of a globular cluster, M28 (Lyne et al. 1987), and in the next few years a total of 34 pulsars were found in globular clusters. These searches were successful because only a few dozen telescope pointings were required and the necessary computations were relatively modest. Most of these discoveries were MSPs, the majority of which were in binary systems, supporting the spinup hypothesis. While these MSPs provided much information about stellar 
interactions within clusters and provided a number of interesting systems to study, they could not be used for their great intrinsic precision as clocks since they were by no means in inertial frames and they gave no indication of how widespread the MSP phenomenon might be in the Galaxy. See Lyne (1994) for a review of globular-cluster searches which will not be discussed further here.

\section{New All Sky Surveys}

By 1992.0, only 5 MSPs were known outside the globular-cluster system, but this figure has now (1994.5) risen to 29. The reasons for this significant advance are two-fold. Firstly the discovery of PSR B1257+12 by Wolszczan \& Frail (1992) at high galactic latitude suggested that MSPs were more isotropic than normal pulsars (Johnston \& Bailes 1991), so encouraging searches away from the galactic plane, where the earlier limited searches had been conducted. Secondly, computing resources available to astronomers had increased by about 2 orders of magnitude over the decade since PSR B1937+21 was discovered. Groups at the three main observatories of Arecibo, Parkes and Jodrell Bank have been active in the recent discoveries. There are also two other surveys of the northern hemisphere being carried out by the Princeton group, using telescopes at Cambridge and Green Bank.

The groups active at Arecibo all used essentially the same observing system, consisting of receivers using an $8 \mathrm{MHz}$ bandwidth centered on $430 \mathrm{MHz}$. The surveys at Parkes and Jodrell Bank were similar, using cryogenic receivers centered on 436 and $410 \mathrm{MHz}$ respectively. Filterbanks were used in all cases and the systems are summarized in Table 1.

The sensitivities of these surveys are complicated functions of pulsar period and dispersion measure (DM). For periods in excess of $10 \mathrm{~ms}$ and for zero DM, the Arecibo and Parkes surveys have sensitivities of 0.9 and $3 \mathrm{mJy}$ on cold sky (i.e., at high galactic latitude). However, even for zero DM, instrumental broadening results in reduction in sensitivity by factors of between about 4 and 10 for objects with period of $1 \mathrm{~ms}$. For higher dispersion measures or shorter periods the sensitivity reduction is even greater. See Camilo (1994) for a fuller technical review of these searches.

Between them, the whole of the sky is being covered by the 3 main surveys. Roughly speaking, the Parkes survey is scheduled to cover the whole sky south of the Equator, the Arecibo experiments to encompass declinations between 0 and +35 degrees while the Jodrell Bank survey covers the region north of about +30 degrees. These surveys are all still in progress but have already proved to be most successful. So far, a total of 29 pulsars have been found and are summarised in Tables 2 and 3.21 of the 29 
TABLE 1. Parameters of 5 Surveys for Millisecond Pulsars.

\begin{tabular}{lccccc}
\hline & Arecibo & Jodrell Bank & Parkes & Cambridge & Green Ban \\
\hline Telescope & $305-\mathrm{m}$ & $76-\mathrm{m}$ & $64-\mathrm{m}$ & $36000-\mathrm{m}^{2}$ & $43-\mathrm{m}$ \\
Frequency (MHz) & 430 & 408 & 436 & 81.5 & 370 \\
Integration Time (sec) & 32.8 & 314 & 157 & 100 & 134 \\
Bandwidth (MHz) & $2 \times 8$ & $2 \times 8$ & $2 \times 32$ & 1 & $2 \times 40$ \\
Channel Width (MHz) & 0.25 & 0.125 & 0.125 & 0.004 & 0.078 \\
Sampling Rate (kHz) & 4.0 & 3.3 & 3.3 & 1.3 & 4 \\
System Temp (K) & 62 & 50 & 55 & - & - \\
Min flux density (mJy) & 0.9 & 4 & 3 & 90 & 10 \\
Area of Sky (deg $\left.{ }^{2}\right)$ & 12700 & 10300 & 20600 & 20000 & 20600 \\
No of beams in area & 582000 & 22000 & 44000 & 17000 & 11300 \\
Area surveyed to 1994.5 $\left(\mathrm{deg}^{2}\right)$ & 3000 & 3000 & 18000 & $?$ & $?$ \\
Fast pulsars found & 11 & 1 & 16 & 0 & 0 \\
\hline
\end{tabular}

objects are in circular orbits with low-mass companions, consistent with the binary accretion process being responsible for the rapid pulsar rotation. Of the 11 pulsars discovered at Arecibo, 5 were discovered in searches prior to 1992 , and 5 in the surveys described above. The eleventh, PSR J0751+18, like PSR B1953+29, was found in a survey of the error boxes of a number of gamma-ray sources. It is not clear whether either of these sources is in fact related to a gamma-ray source.

A number of notable pulsars lie within these lists of discoveries. Firstly PSR $1257+12$ is an object which appears to have at least 3 bodies of planetary mass in orbit around it (Wolszczan \& Frail 1992). Another, PSR J04374715 is a 5.75 millisecond pulsar in a circular 5.7-day orbit with a companion of $\sim 0.14 \mathrm{M}_{\odot}$ (Johnston et al. 1993). It is by far the brightest of all known MSPs and high-quality single pulses have been observed, it is also the closest known millisecond pulsar to the Earth, with a distance of about $150 \mathrm{pc}$. The proximity of the binary system to Earth offers new opportunity for studies at all wavelengths. Indeed, the companion has been optically identified (Bell, Bailes \& Bessell 1993), and X-rays have been detected from the surface of the neutron star (Becker \& Trümper 1993). Two of these pulsars show eclipses by the atmosphere of the companion stars, PSRs B1957+20 and J2052-08. The former was the first such system to be found and optically identified (Fruchter, Stinebring \& Taylor 1988). Occulted by greater periods at lower frequency, it seems that the occultation is due to absorption in a wind from the companion star which is being ablated by high-energy radiation from the pulsar (Ruderman, Shaham \& 
TABLE 2. Millisecond Pulsars Discovered at Arecibo.

\begin{tabular}{lrrrrrrrrrc}
\hline \multicolumn{1}{c}{ PSR } & $\begin{array}{c}P_{\text {rot }} \\
\mathrm{ms}\end{array}$ & $\begin{array}{c}P_{\text {orb }} \\
\text { days }\end{array}$ & $\mathrm{e}$ & $\begin{array}{c}M_{\text {min }} \\
\mathrm{M}_{\odot}\end{array}$ & $\begin{array}{c}d \\
\mathrm{kpc}\end{array}$ & $\begin{array}{c}z \\
\mathrm{kpc}\end{array}$ & $\begin{array}{c}\log (\tau) \\
\log (\mathrm{yr})\end{array}$ & $\begin{array}{c}\log (B) \\
\log (\mathrm{G})\end{array}$ & $\begin{array}{c}V_{\mathrm{t}} \\
\mathrm{km} \mathrm{s}^{-1}\end{array}$ & $\begin{array}{c}\text { Dis } \\
\text { Ref }\end{array}$ \\
\hline $\mathrm{J} 0751+18$ & 3.48 & 0.3 & ${ }^{*} 0.0$ & .13 & 2.00 & .72 & & & & 1 \\
$\mathrm{~J} 1025+10$ & 16.45 & 7.8 & ${ }^{*} 0.0$ & .72 & & & & & & 2 \\
$\mathrm{~B} 1257+12$ & 6.22 & & & & .62 & .60 & 8.95 & 8.93 & 276 & 3 \\
$\mathrm{~J} 1713+0747$ & 4.57 & 67.8 & .000075 & .28 & .89 & .38 & 9.95 & 8.30 & 31 & 4 \\
$\mathrm{~B} 1855+09$ & 5.36 & 12.3 & .000022 & .25 & 1.00 & .05 & 9.68 & 8.49 & 28 & 5 \\
& & & & & & & & & & \\
$\mathrm{~B} 1937+21$ & 1.56 & & & & 3.58 & -.02 & 8.36 & 8.62 & 10 & 6 \\
$\mathrm{~B} 1953+29$ & 6.13 & 117.3 & .000330 & .18 & 5.39 & .04 & 9.52 & 8.63 & & 7 \\
$\mathrm{~B} 1957+20$ & 1.61 & 0.4 & .000040 & .02 & 1.53 & -.13 & 9.17 & 8.23 & 206 & 8 \\
$\mathrm{~J} 2019+2425$ & 3.93 & 76.5 & .000111 & .32 & .91 & -.11 & 9.95 & 8.26 & 102 & 9 \\
$\mathrm{~J} 2317+1439$ & 3.44 & 2.5 & .000001 & .18 & 1.89 & -1.27 & 10.3 & 8.00 & & 10 \\
& & & & & & & & & & \\
$\mathrm{~J} 2322+2057$ & 4.81 & & & & .78 & -.47 & 8.89 & 8.34 & 94 & 9 \\
\hline
\end{tabular}

* - The eccentricity of these systems is small, but not yet measured.

Reference keys:

1: Lundgren, Zepka \& Cordes 1995; 2: Camilo 1995; 3: Wolszczan \& Frail 1992; 4: Foster, Wolszczan \& Camilo 1993; 5: Stokes, Taylor \& Dewey 1985; 6: Backer et al. 1982; 7: Boriakoff, Buccheri \& Fauci 1983; 8: Fruchter, Stinebring \& Taylor 1988; 9: Nice, Taylor \& Fruchter 1993; 10: Camilo, Nice \& Taylor 1993.

Tavani 1989; Rasio, Shapiro \& Teukolsky 1989). This is supported by the observation that the optical brightness of the companion star varies with orbital phase, being brightest as the side illuminated by the pulsar faces the Earth (Kulkarni, Djorgovski \& Fruchter 1988). Three other known occulting low-mass systems are all in globular clusters. Both PSR B1957+20 and PSR J0437-4715 show $\mathrm{H} \alpha$ wind nebulae where the pulsar high-energy radiation interacts with the interstellar medium (Kulkarni \& Hester 1988; Bell et al. 1995).

\section{Statistical Overview}

The findings of the two main surveys are roughly consistent with the same space density of MSPs in the local galactic plane. Taking into account the regions of sky so far covered, we find that the Parkes survey is discovering pulsars at a rate of $1 \mathrm{MSP} / 1000 \mathrm{deg}^{2}$ above the limiting flux density of $3 \mathrm{mJy}$. Because of the greater sensitivity, the Arecibo surveys are finding 
TABLE 3. Millisecond Pulsars Discovered at Parkes and Jodrell Bank.

\begin{tabular}{|c|c|c|c|c|c|c|c|c|c|c|}
\hline PSR & $\begin{array}{c}P_{\text {rot }} \\
\text { ms }\end{array}$ & $\begin{array}{l}P_{\text {orb }} \\
\text { days }\end{array}$ & e & $\begin{array}{c}M_{\min } \\
\mathbf{M}_{\odot}\end{array}$ & $\begin{array}{c}d \\
\text { kpc }\end{array}$ & $\begin{array}{c}z \\
\mathbf{k p c}\end{array}$ & $\begin{array}{c}\log (\tau) \\
\log (y \mathbf{r})\end{array}$ & $\begin{array}{l}\log (B) \\
\log (G)\end{array}$ & 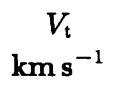 & $\begin{array}{l}\text { Dis } \\
\text { Ref }\end{array}$ \\
\hline \multicolumn{11}{|c|}{ Parkes Millisecond Pulsars } \\
\hline J0034-0534 & 1.88 & 1.6 & $<.0001$ & .14 & .98 & -.91 & 9.64 & 8.04 & & 1 \\
\hline J0437-4715 & 5.76 & 5.7 & .000020 & .14 & .14 & -.09 & 9.20 & 8.76 & 72 & 2 \\
\hline J0613-0200 & 3.06 & 1.2 & $<.000022$ & .13 & 2.19 & -.35 & 9.69 & 8.26 & & 3 \\
\hline J0712-68 & 5.49 & & & & 1.02 & -.40 & & & & 4 \\
\hline J1025-07 & 5.16 & & & & .35 & .23 & & & & 4 \\
\hline J1045-4509 & 7.47 & 4.1 & .000019 & .16 & 3.24 & .69 & 9.79 & 8.58 & & 1 \\
\hline J1455-3330 & 7.99 & 76.2 & .000167 & .26 & .74 & .28 & $>10.3$ & $<8.3$ & 60 & 3 \\
\hline J1604-72 & 14.84 & 6.3 & $*_{0.0}$ & .26 & 1.65 & .43 & & & & \\
\hline J1643-1224 & 4.62 & 147.0 & .000506 & .12 & 4.86 & 1.76 & 9.34 & 8.60 & & 3 \\
\hline J1730-2304 & 8.12 & & & & .51 & .05 & $>9.6$ & $<8.7$ & 22 & 3 \\
\hline J1745-11 & 4.07 & & & & .17 & .03 & & & & \\
\hline J1804-27 & 9.34 & 11 & $* 0.0$ & .20 & 1.17 & -.06 & & & & 4 \\
\hline J2052-08 & 4.51 & 0.099 & $* 0.0$ & .03 & 1.23 & -.63 & & & & \\
\hline J2124-3358 & 4.93 & & & & .24 & -.17 & $>9.1$ & $<8.7$ & & 4 \\
\hline J2129-57 & 3.73 & 6.6 & ${ }^{*} 0.0$ & .13 & 2.54 & -1.76 & & & & \\
\hline J2145-0750 & 16.05 & 6.8 & .000021 & .43 & .50 & -.34 & $>10.1$ & $<8.8$ & 31 & 1 \\
\hline \multicolumn{11}{|c|}{ Jodrell Bank/Caltech Millisecond pulsars } \\
\hline $\mathrm{J} 0218+4232$ & 2.32 & 2.0 & $<0.00002$ & .17 & 5.85 & -1.76 & 9.58 & 8.18 & & \\
\hline $\mathrm{J} 1012+5307$ & 5.26 & 0.6 & ${ }^{*} 0.0$ & .11 & .53 & .41 & & & & 5 \\
\hline
\end{tabular}

Reference keys:

1: Bailes et al. 1994; 2: Johnston et al. 1993; 3: Lorimer et al. 1995; 4 :Lorimer 1994; 5: Nicastro et al. 1995.

pulsars at a rate of about $4 \mathrm{MSP} / 1000 \mathrm{deg}^{2}$ above the limiting flux density of $0.9 \mathrm{mJy}$. We would expect the observed surface density of pulsars observed by Arecibo to exceed that observed by Parkes by a factor of 3.3 if the pulsars are distributed in an essentially planar population or by a factor of 6 for an isotropic distribution. The observed factor of 4 is within this range and is consistent with what can be seen from the distribution of the pulsars in Tables 3 and 4, that MSPs clearly have a large scale height, albeit with 


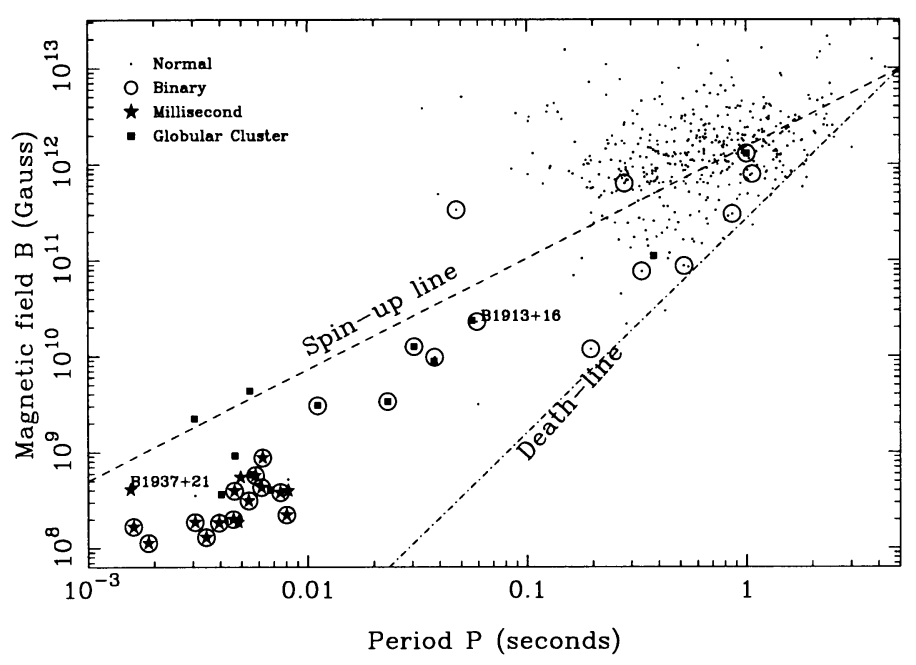

Figure 1. Pulsar magnetic field against rotational period for all known pulsars with period derivative measurements.

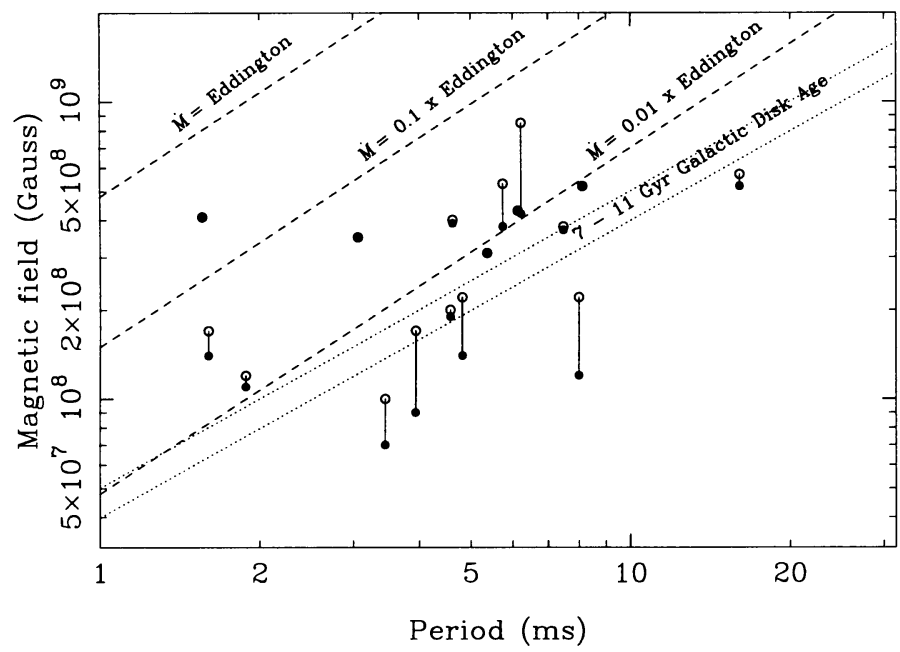

Figure 2. Pulsar magnetic field against rotational period for millisecond pulsars. The open symbols are the fields derived from the measured values of period derivative while the filled ones are derived after correction for the Shklovskii effect (see text). The dotted lines are isochrones delimiting the likely age of the Galaxy.

some concentration towards the Galactic Plane (Lorimer 1995).

All the MSPs have small surface magnetic fields and high ages, as determined from their period derivatives in the normal way (Manchester \& Taylor 1977). Fig. 1 shows their position in the $B-P$ diagram relative to the rest of the pulsar population. However, it has been pointed out by 


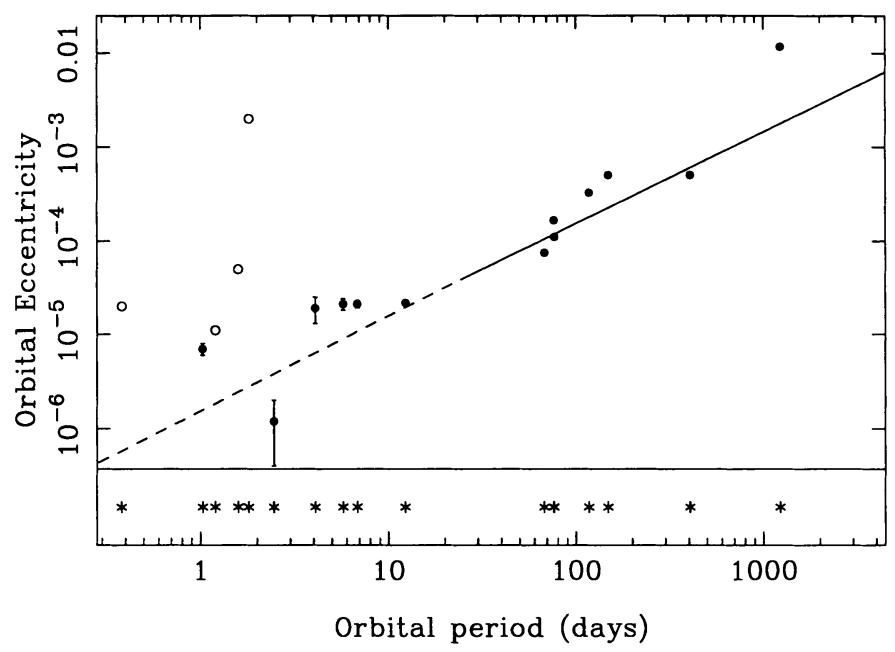

Figure 3. Orbital eccentricity against orbital period for low-mass pulsar binary systems. The open symbols represent orbits with only upper limits to the eccentricity measurement. Along the bottom of the diagram is the distribution in orbital period.

Camilo et al. (1994) that the observed values of derivative, $\dot{P}$, are contaminated by the Shklovskii effect (Shklovskii 1970) which arises from the pulsar transverse speed, $V_{t}$, and increases them by an amount $\Delta \dot{P}=P \times V_{t}^{2} / c d$, where $d$ is the distance and $c$ is the velocity of light. After correction for this effect, the ages of several pulsars exceed $10^{10}$ years, in excess of the age of the Galaxy as can be seen in Fig. 2 which displays the lower lefthand corner of Fig. 1 together with corrected values of $B$ for those pulsars for which estimates of their transverse speeds are available (Lorimer 1994). The great ages suggest that either the present periods are similar to those at birth, immediately after spin-up, or else their magnetic fields may have decayed.

There is good evidence that the fast rotation rates are due to processing in binary systems. The fact that the large majority are in circular orbit with low-mass companions supports this hypothesis as does the loose relationship between the spin period and orbital period predicted for binary pulsars (Bailes et al. 1994), similar to the Corbet diagram (Corbet 1984) for high-mass X-ray binaries. Moreover, the evolution of the mass-exchanging systems in which spin up takes place is expected to result in a relation between the final pulsar orbital period and its white-dwarf companion mass (Joss, Rappaport \& Lewis 1987). Despite the uncertainty in determining these masses due to the usually unknown orbital inclination, most pulsars in circular low-mass systems do obey this law.

Expected eccentricities are expected to be $\sim 10^{-43}$ or less, due to the 


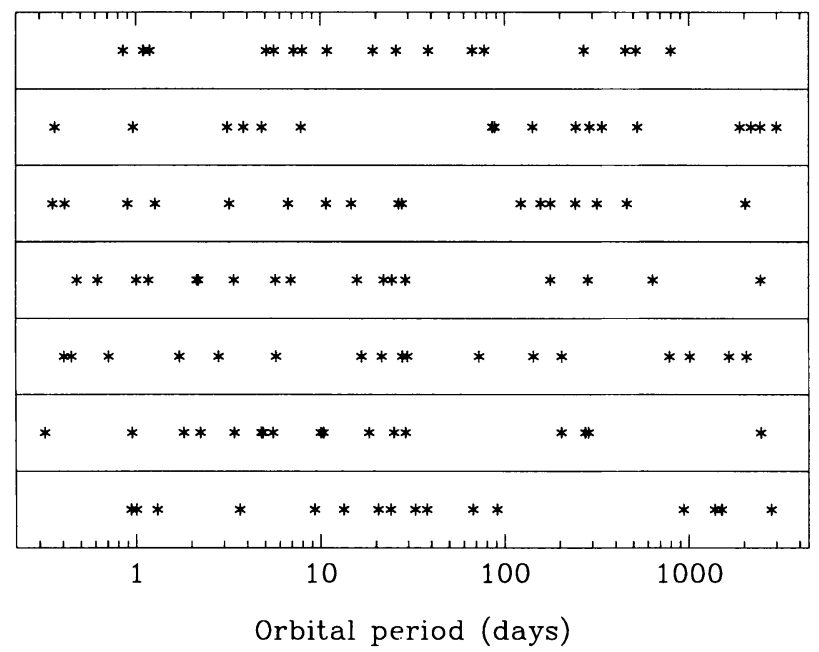

Figure 4. The orbital period distributions resulting from seven consecutive Monte Carlo simulations obtained assuming uniform probability between 0.3 and 3000 days. Most of these show gaps of similar width to that observed in Fig. 3.

circularising effect of the accretion process (Phinney 1993). These values are tiny compared with the observed values which all lie between $10^{-6}$ and $10^{-2}$. Phinney suggests that this might arise from convection in the giant star prior to termination of accretion. He predicts a linear relationship between the eccentricity and orbital period, which is shown in Fig. 3 together with the observed values which follow the prediction extremely well (Lorimer et al. 1995). This provides further good circumstantial evidence for the spin-up scenario for these objects.

The distribution of orbital period is also shown at the bottom of Fig. 3, showing a gap over a factor of about 6 , centered on 30 days (Camilo 1994). In the context of the orbital period/companion mass relationship (Joss, Rappaport \& Lewis 1987), this would imply a gap in the masses of the companions. A simple Monte Carlo simulation demonstrates that the gap is not significant. Fig. 4 shows 7 successive simulations which assume a uniform probability distribution of 17 periods between 0.3 and 3000 days. In each of these, there is a gap over a similar factor somewhere in the distribution. Clearly, much larger numbers of systems will be required to establish whether the proposed gap in orbital period and hence in companion mass really exists. 


\section{Conclusion}

The surveys discussed above have been remarkably successful at detecting these very weak objects. The MSPs show a large range of extreme astrophysical phenomena and, as clocks, they show incredible rotation stability and promise to act as superb probes of gravitation fields. However, despite these successes, the surveys have very poor sensitivity to any MSPs which have rotational periods of a millisecond or less or any which may be highly accelerated in close or massive binary systems. In the next 10 years or so, computing resources are expected to increase enough to allow us to find out whether such exotic systems exist.

\section{References}

Alpar, M.A. et al. 1982, Nat 300, 728

Backer, D. C. et al. 1982, Nat 300, 615

Bailes, M. et al. 1994, ApJ 425, L41

Becker, W. \& Trümper, J. 1993, Nat 365, 528

Bell, J.F., Bailes, M. \& Bessell, M.S. 1993, Nat 364, 603

Bell, J.F. et al. 1995, ApJ 440, L81

Boriakoff, V., Buccheri, R. \& Fauci, F. 1983, Nat 304, 417

Camilo, F. 1994, In: The Lives of the Neutron Stars (NATO ASI Series), M.A. Alpar, Ü. Kızıloğlu \& J. van Paradijs (Eds.), Kluwer (Dordrecht), p. 243

Camilo, F. 1995, PhD thesis, Princeton University

Camilo, F., Nice, D.J. \& Taylor, J.H. 1993, ApJ 412, L37

Camilo, F., Thorsett, S.E. \& Kulkarni, S.R. 1994, ApJ 421, L15

Corbet, R.H.D. 1984, A\&A 141, 91

Fabian, A.C., Pringle, J.E. \& Rees, M.J. 1975, MNRAS 172, 15P

Foster, R.S., Wolszczan, A. \& Camilo, F. 1993, ApJ 410, L91

Fruchter, A.S., Stinebring, D.R. \& Taylor, J.H. 1988, Nat 333, 237

Johnston, S. \& Bailes, M. 1991, MNRAS 252, 277

Johnston, S. et al. 1993, Nat 361, 613

Joss, P.C., Rappaport, S. \& Lewis, W. 1987, ApJ 319, 180

Kulkarni, S.R. \& Hester, J.J. 1988, Nat 335, 801

Kulkarni, S.R., Djorgovski, S. \& Fruchter, A.S. 1988, Nat 334, 504

Lorimer, D.R. 1994, PhD thesis, The University of Manchester

Lorimer, D.R. 1995, these proceedings

Lorimer, D.R. et al. 1995, ApJ 439, 933

Lundgren, S.C., Zepka, A.F. \& Cordes, J.M. 1995, ApJ (submitted)

Lyne, A.G. 1994, in Millisecond Pulsars - A Decade of Surprises, A. Fruchter, M. Tavani,

\& D.C. Backer (Eds.), Astronomical Society of the Pacific, (in press)

Lyne, A.G. et al. 1987, Nat 328, 399

Manchester, R.N. \& Taylor, J.H. 1977, Pulsars, Freeman (San Francisco)

Nicastro, L. et al. 1995, MNRAS (in press)

Nice, D.J., Taylor, J.H. \& Fruchter, A.S. 1993, ApJ 402, L49

Phinney, E.S. 1993, Phil. Trans. Roy. Soc. A341, 39

Rasio, F.A., Shapiro, S.L. \& Teukolsky, S.A. 1989, ApJ 342, 934

Ruderman, M., Shaham, J. \& Tavani, M. 1989, ApJ 336, 507

Shklovskii, I.S. 1970, SvA 13, 562

Stokes, G.H., Taylor, J. \& Dewey, R.J. 1985, ApJ 294, L21

Wolszczan, A. \& Frail, D.A. 1992, Nat 355, 145 